\title{
Erratum to: Functional Characteristics and Molecular Mechanism of a New scFv Antibody Against A $\beta 42$ Oligomers and Immature Protofibrils
}

\author{
Yuan Zhang • Yuanhong Sun • Yangyang Huai • Ying-Jiu Zhang
}

Published online: 15 December 2014

(C) Springer Science+Business Media New York 2014

\section{Erratum to: Mol Neurobiol}

DOI 10.1007/s12035-014-8910-7

Figures $2 \mathrm{c}$ and $7 \mathrm{c}$ of the original articles unfortunately contain mistakes. The authors would like to correct these figures and are now elaborated as follows:

(1) In Fig. 2c, the names of the two lanes should be swapped left-right. In the second line from the bottom of its caption, the word "left" should be "right."

(2) A $342-Q 26$ in Fig. 7c should be A $\beta 42-\mathrm{N} 27$

Correct Figs. 2 and 7 are hereby published.

The online version of the original article can be found at http://dx.doi.org/ 10.1007/s12035-014-8910-7.

Y. Zhang $\cdot$ Y. Sun $\cdot$ Y. Huai $\cdot$ Y.-J. Zhang $(\bowtie)$

Key Laboratory for Molecular Enzymology and Engineering of Ministry of Education, Jilin University, 130012 Changchun, China

e-mail: yingjiu@jlu.edu.cn

\section{Y.-J. Zhang}

National Engineering Laboratory for AIDS Vaccine, Jilin University,

130012 Changchun, China

\section{Y.-J. Zhang}

School of Life Science, Jilin University, 130012 Changchun, China 


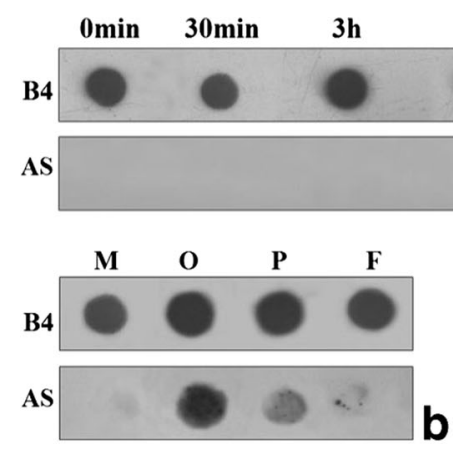

Fig. 2 Dot blot and Western blot analyses for the binding specificities of $\mathrm{scFv}$ AS to various $\mathrm{A} \beta 42$ species. Dots of $\mathrm{A} \beta 42$ monomers after incubation for an indicated period of time ( 0 and $30 \mathrm{~min} ; 3,6,12$, and $24 \mathrm{~h}$ ) a or of A 342 monomers, oligomers, protofibrils, and fibrils $\mathbf{b}$ were probed with B4 and scFv AS, respectively, and then were detected with HRP-
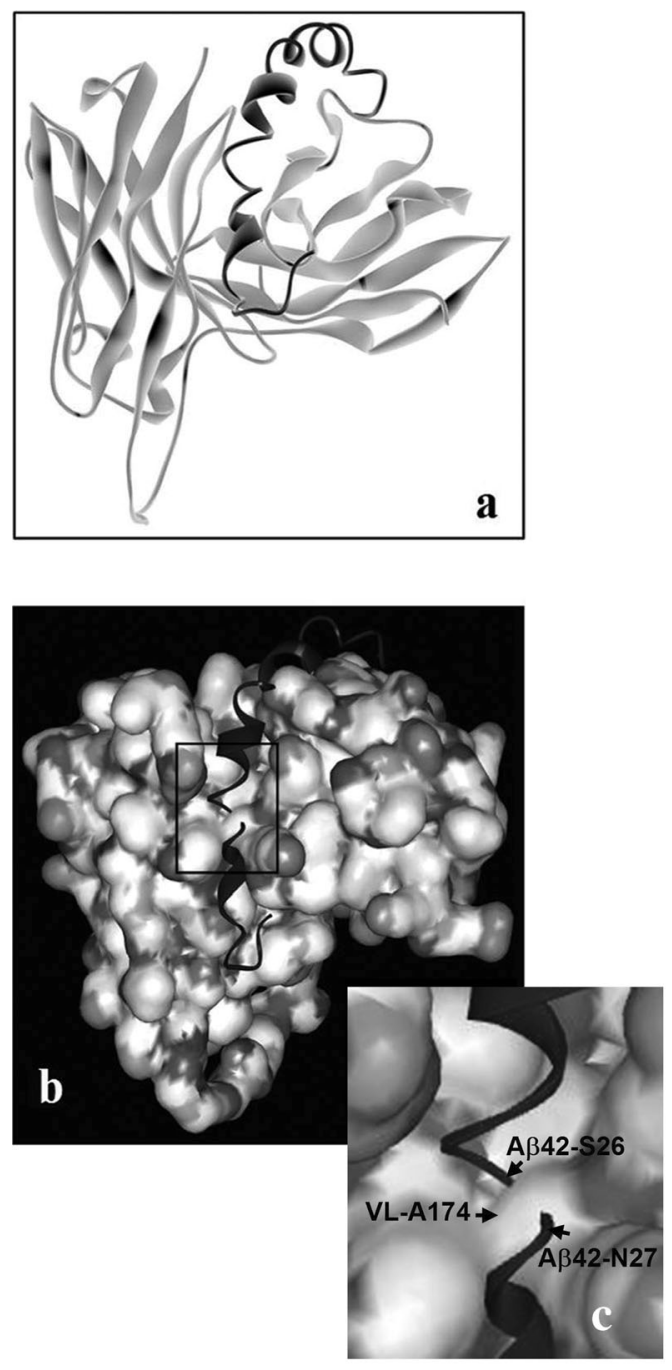

Fig. 7 Molecular docking model of A $\beta 42$ monomer to scFv AS. a Ribbon display mode showing A $\beta 42$ in dark black, scFv AS in light black. b Surface representation of the model. c Close-up view of VL-Ala174, A $342-S e r 26$, and Aß42-Asn27 as those in b. d The hydrogen bonding 12h 24h

\section{(1)}

a

C

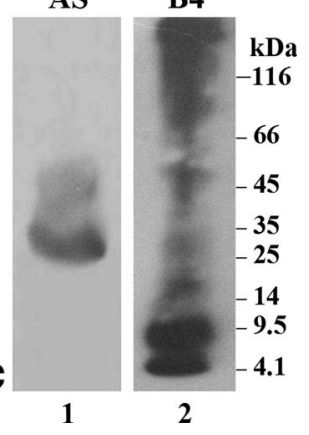

conjugated IgG and visualized with ECL chemiluminescence kit. Detection of $\mathrm{scFv}$ AS binding to various $\mathrm{A} \beta 42$ species ranging from $\mathrm{A} \beta 42$ monomers to fibrils by Western blot c. B4 served as positive controls [top row in a and $\mathbf{b}$ or right lane in c]. $M$ monomers, $O$ oligomers, $P$ protofibrils, $F$ fibrils

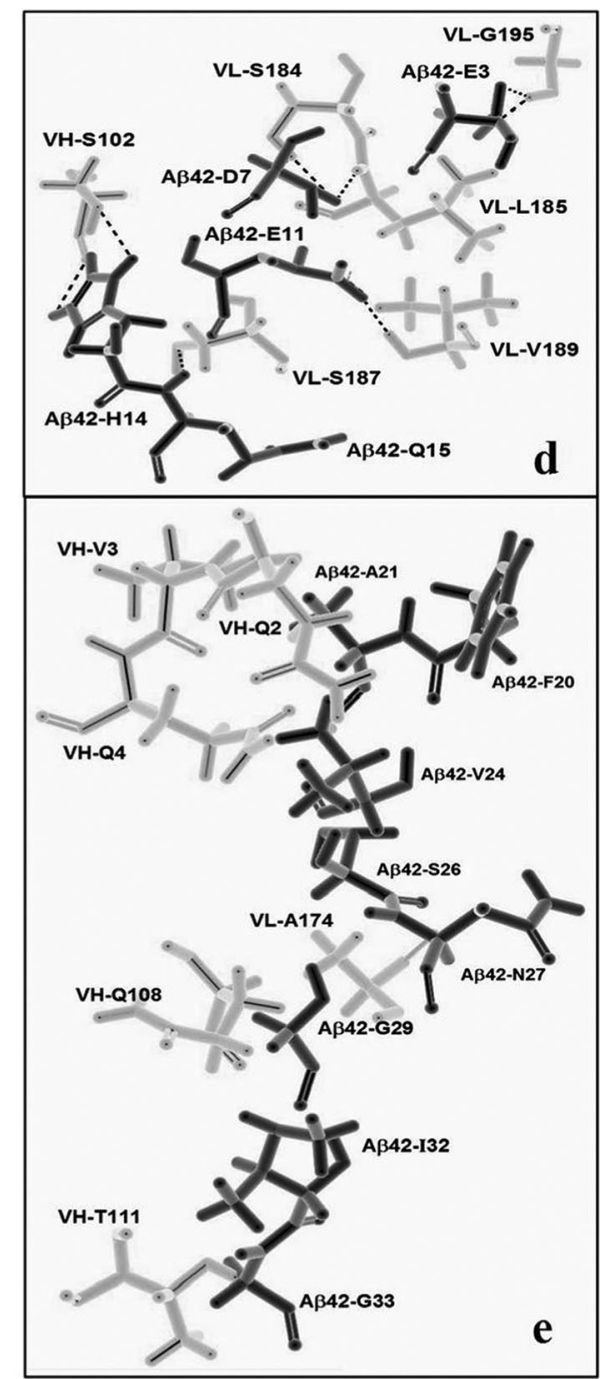

interactions (dashed lines) between $\mathrm{scFv} \mathrm{AS}$ and the $\mathrm{N}$-terminal region (residues 1-15) of A $\beta 42$ monomer. e The hydrophobic bonding interactions between $\mathrm{scFv}$ AS and the central region (residues 20-33) of $\mathrm{A} \beta 42$ monomerk 